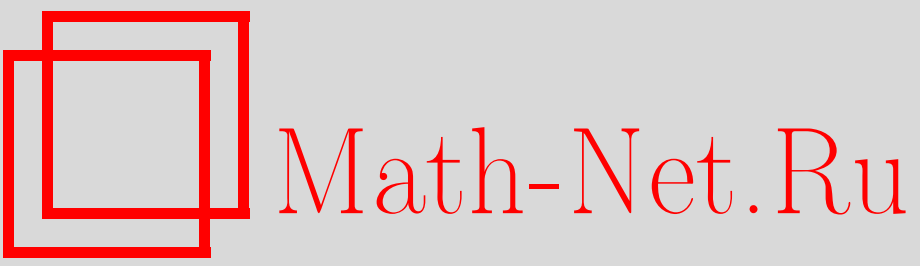

Е. И. Яблокова, О собственных колебаниях тела с концентрированными массами критической плотности, расположенными непериодически на поверхности, УМH, 2003, том 58, выпуск 6, 171-172

DOI: https://doi.org/10.4213/rm692

Использование Общероссийского математического портала Math-Net.Ru подразумевает, что вы прочитали и согласны с пользовательским соглашением

http://www.mathnet.ru/rus/agreement

Параметры загрузки:

IP : 3.89 .185 .249

26 апреля 2023 г., 14:59:21 


\title{
О СОБСТВЕННЫХ КОЛЕБАНИЯХ ТЕЛА \\ С КОНЦЕНТРИРОВАННЫМИ МАССАМИ \\ КРИТИЧЕСКОЙ ПЛОТНОСТИ, РАСПОЛОЖЕННЫМИ НЕПЕРИОДИЧЕСКИ НА ПОВЕРХНОСТИ
}

\author{
Е. И. ЯьлоковА
}

Постановка задачи. Мы рассматриваем спектральную задачу для стационарной линейной системы теории упругости с быстро меняющимся типом граничных условий в области с большим количеством концентрированных масс около границы. Не предполагая периодичности микроструктуры области, автор рассматривает случай, когда плотность концентрированных масс имеет критическое значение. В статьях [1] и [2] рассматриваются похожие задачи в областях с концентрированными массами, расположенными периодически вдоль границы, в [3] - задача с непериодически расположенными “легкими” концентрированньми массами.

Пусть $\Omega \subset\left\{x \in \mathbb{R}^{d}, x_{d}>0\right\}$ - ограниченная область с гладкой границей $\partial \Omega, d \geqslant 3$. Полагаем, что $\partial \Omega=\overline{\Gamma_{1}} \cup \overline{\Gamma_{2}}$, где $\Gamma_{2} \subset\left\{x_{d}=0\right\}, \overline{\Gamma_{2}}=\overline{\Gamma_{\varepsilon}} \cup \overline{\gamma_{\varepsilon}}$, при этом $\Gamma_{\varepsilon}=\bigcup_{n=1}^{N_{\varepsilon}} \Gamma_{\varepsilon}^{n}$, где $\operatorname{diam}\left(\Gamma_{\varepsilon}^{n}\right) \leqslant 2 \varepsilon$, а расстояние между двумя множествами $\Gamma_{\varepsilon}^{n}$ много больше $\varepsilon ; \varepsilon$ - малшй положительньй параметр. Рассмотрим спектральную задачу

$$
\left\{\begin{array}{l}
\mathscr{L}_{k}\left[u_{\varepsilon}^{K}\right]+\lambda_{\varepsilon}^{K} \rho_{\varepsilon}(x) u_{\varepsilon}^{K^{k}}=0 \text { в } \Omega, k=1, \ldots, d, K=1,2, \ldots, \\
\left(u_{\varepsilon}^{I}, u_{\varepsilon}^{J}\right)_{\left[L_{2, \rho_{\varepsilon}}(\Omega)\right]^{d}} \equiv \sum_{k=1}^{d} \int_{\Omega} u_{\varepsilon}^{I^{k}} \rho_{\varepsilon} u_{\varepsilon}^{J^{k}} d x=\delta_{I J}, \\
u_{\varepsilon}^{K}=0 \text { на } \Gamma_{\varepsilon} \cup \Gamma_{1}, \quad \sigma_{k}\left(u_{\varepsilon}^{K}\right) \equiv a_{k l}^{i j}(x) \frac{\partial u_{\varepsilon}^{K^{l}}}{\partial x_{i}} \nu_{j}=0 \text { на } \gamma_{\varepsilon},
\end{array}\right.
$$

по повторяющимся индексам предполагается суммирование от 1 до $d$. Здесь $\mathscr{L}_{k}\left[u_{\varepsilon}^{K}\right] \equiv$ $\frac{\partial}{\partial x_{j}}\left(a_{k l}^{i j}(x) \frac{\partial u_{\varepsilon}^{K^{l}}}{\partial x_{i}}\right), \nu=\left(\nu_{1}, \ldots, \nu_{d}\right)$ - внешняя нормаль к границе $\partial \Omega ; u_{\varepsilon}^{K}=\left(u_{\varepsilon}^{K^{1}}, \ldots, u_{\varepsilon}^{K^{d}}\right)$, $\delta_{I J}$-символ Кронекера. Такжепредполагается выполнение условий $a_{k l}^{i j}(x)=a_{l k}^{j i}(x)=a_{i l}^{k j}(x)$ и $\varkappa_{1} \xi_{k i} \xi_{k i} \leqslant a_{k l}^{i j}(x) \xi_{k i} \xi_{l j} \leqslant \varkappa_{2} \xi_{k i} \xi_{k i}$, где $\varkappa_{1}, \varkappa_{2}=$ const $>0, x \in \Omega$, а $\left\{\xi_{k i}\right\}$ - действительные симметрические матрицы, $a_{k l}^{i j}$ - ограниченные измеримые функции в $\Omega$.

Обозначение $\left[H^{1}\left(\Omega, \Gamma_{\varepsilon} \cup \Gamma_{1}\right)\right]^{d}$ мы используем для множества вектор-функций из соболевского пространства $\left[H^{1}(\Omega)\right]^{d}$ с нулевьм следом на $\Gamma_{\varepsilon} \cup \Gamma_{1}$.

Собственные значения $\left\{\lambda_{\varepsilon}^{K}, K=1,2, \ldots\right\}$ занумерованы в порядке неубывания, т.е. $0<$ $\lambda_{\varepsilon}^{1} \leqslant \cdots \leqslant \lambda_{\varepsilon}^{K} \leqslant \cdots$, и повторяются с учетом кратности. Мы рассматриваем специальную плотность вида $\rho_{\varepsilon}(x)=\left\{\begin{array}{ll}1 & \text { в } \Omega \backslash B_{\varepsilon}, \\ \varepsilon^{-2} & \text { в } \overline{\Omega \cap B_{\varepsilon}},\end{array}\right.$ где $B_{\varepsilon}=\bigcup_{n=1}^{N_{\varepsilon}} B_{\varepsilon}^{n}$, а $B_{\varepsilon}^{n}=\left\{\left(x_{1}-\eta_{1}^{n}\right)^{2}+\cdots+\right.$ $\left.\left(x_{d}-\eta_{d}^{n}\right)^{2}<\varepsilon^{2}\right\}$, при этом точка $p_{\varepsilon}^{n}=\left(\eta_{1}^{n}, \ldots, \eta_{d}^{n}\right)$ принадлежит $\Gamma_{\varepsilon}^{n}, n=1, \ldots, N_{\varepsilon}$, таким образом, что $\overline{B_{\varepsilon}^{n}} \cap \partial \Omega=\Gamma_{\varepsilon}^{n}$. Множества $B_{\varepsilon}^{n}$ назовем концентрированнылми массами; число концентрированных масс $N_{\varepsilon}=O\left(|\ln \varepsilon|^{\alpha}\right), \alpha>0$.

Переход к локальным координатам. Для изучения предельного поведения собственных значений задачи (1) перейдем к локальным координатам $\xi=\frac{x-p_{\varepsilon}^{n}}{\varepsilon}$. Обозначим $\mathbb{R}^{d^{+}}$полупространство $\left\{\xi \in \mathbb{R}^{d}: \xi_{d}>0\right\}$.

Пусть полушар $B_{1}=\left\{|\xi|<1, \xi_{d}>0\right\}$ является образом $B_{\varepsilon}^{n}$ в локальных координатах $\xi$, аналогично $\Gamma_{\xi}=\left\{|\xi|<1, \xi_{d}=0\right\}-$ образ $\Gamma_{\varepsilon}^{n}, \gamma_{\xi}=\left\{\xi_{d}=0\right\} \backslash \overline{\Gamma_{\xi}}$.

Пространство $\left[H\left(\mathbb{R}^{d^{+}}, \Gamma_{\xi}\right)\right]^{d}$ определим как пополнение множества вектор-функций из пространства $\left[C^{\infty}\left(\mathbb{R}^{d^{+}}\right)\right]^{d}$, обращающихся в нуль в окрестности $\Gamma_{\xi}$, по следующей норме: 
$\|v\|_{\left[H\left(\mathbb{R}^{d^{+}}\right)\right]^{d}}^{2}=\sum_{k=1}^{d} \int_{\mathbb{R}^{d^{+}}}\left(\varepsilon^{2}\left|v^{k}\right|^{2}+\left|\nabla_{\xi} v^{k}\right|^{2}\right) d \xi$.

Рассмотрим следующую спектральную задачу:

$$
\left\{\begin{array}{l}
\widetilde{L}_{k}\left[U_{0}^{K}\right]+\Lambda_{0}^{K} \chi_{B_{1}} U_{0}^{K^{k}}=0 \text { в } \mathbb{R}^{d^{+}}, \quad K=1,2, \ldots, k=1, \ldots, d, \\
\mathscr{L}_{k}\left[u_{0}^{K}\right]+\Lambda_{0}^{K} u_{0}^{K^{k}}=0 \text { в } \Omega, \\
U_{0}^{K} \rightarrow 0 \text { при }|\xi| \rightarrow \infty, \xi_{d}>0, \quad U_{0}^{K}=0 \text { на } \Gamma_{\xi}, \\
\widetilde{\sigma}_{k}\left(U_{0}^{K}\right)=-a_{k l}^{i d}(\varepsilon \xi) \frac{\partial U_{0}^{K^{l}}}{\partial \xi_{i}}=0 \text { на } \gamma_{\xi}, \\
\sigma_{k}\left(u_{0}^{K}\right)=0 \text { на } \partial \Omega,
\end{array}\right.
$$

где $\widetilde{\mathscr{L}}_{k}\left[U_{0}^{K}\right] \equiv \frac{\partial}{\partial \xi_{j}}\left(a_{k l}^{i j}(\varepsilon \xi) \frac{\partial U_{0}^{K^{l}}}{\partial \xi_{i}}\right), \chi_{B_{1}}-$ характеристическая функция множества $B_{1}$. Собственные функции $\left\{U_{0}^{K} \in\left[H\left(\mathbb{R}^{d^{+}}\right)\right]^{d}, K=1,2, \ldots\right\}$ образуют ортогональньй базис в $\left[L_{2}\left(B_{1}\right)\right]^{d}$, a $\left\{u_{0}^{K} \in\left[H^{1}(\Omega)\right]^{d}, K=1,2, \ldots\right\}-$ в $\left[L_{2}(\Omega)\right]^{d}$. Собственные значени $\left\{\Lambda_{0}^{K}, K=1,2, \ldots\right\}$ занумерованы в порядке неубьвания и повторяются в соответствии с их кратностью.

Основной результат. Используя спектральную теорему о предельном поведении спектров положительных самосопряженных компактных операторов, определенных на различных гильбертовых пространствах, зависящих от малого параметра (см. теорему 1.9 гл. III в [4]), доказываем следующую оценку для разности собственных значений задач (1) и (2).

Теорема 1. Для соответствующих собственных значений $\lambda_{\varepsilon}^{K}$ и $\Lambda_{0}^{K}$ задач (1) и (2) при указанных предположениях имеет место следующая оценка:

$$
\left|\lambda_{\varepsilon}^{K}-\Lambda_{0}^{K}\right| \leqslant C_{K}|\ln \varepsilon|^{\frac{1}{d}-1+\alpha},
$$

где константы $C_{K}$ не зависят от $\varepsilon$.

В заключение автор выражает глубокую признательность Г. А. Чечкину за постановку задачи и внимание к работе.

\section{СПИСОК ЛИТЕРАТУРЫ}

[1] Г. А. Чечкин // УМН. 1995. Т. 50. №4. С. 105-106. [2] M. Lobo, E. Pérez // C. R. Acad. Sci. Paris. Sér. II b. 1999. V. 327. P. 771-776. [3] М. Е. Перес, Г. А. Чечкин, Е. И. Яблокова (Доронина) // УМН. 2002. Т. 57. № 6. С. 195-196. [4] О. А. Олейник, Г.А. Иосифьян, А.С. Шамаев. Математические задачи теории сильно неоднородных упругих сред. М.: МГУ, 1990.

Московский государственный

Принято редколлегией университет им. М. В. Ломоносова 23.09 .2003

E-mail: eidor@rambler.ru, edoron@it.ru 